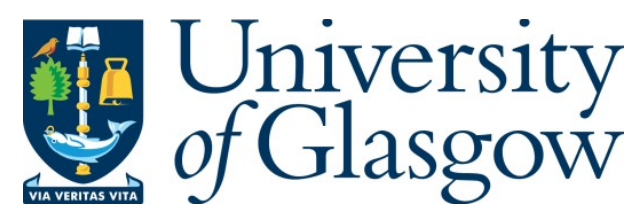

Fitzgibbon, T.A. and Barakos, G.N. (2021) Investigation of wake breakdown in hover using the HMB3 solver. In: AIAA SCITECH 2022 Forum, San Diego, CA \& Virtual, 37 January 2022, ISBN 9781624106316

(doi: $\underline{10.2514 / 6.2022-1320)}$

This is the Author Accepted Manuscript.

There may be differences between this version and the published version. You are advised to consult the publisher's version if you wish to cite from it.

http://eprints.gla.ac.uk/260570/

Deposited on: 13 December 2021

Enlighten - Research publications by members of the University of Glasgow http://eprints.gla.ac.uk 


\title{
Investigation of Wake Breakdown in Hover Using The HMB3 Solver
}

\author{
T. A. Fitzgibbon*, G.N. Barakos ${ }^{\dagger}$ \\ CFD Laboratory, School of Engineering, University of Glasgow, G12 8QQ Glasgow, UK
}

\begin{abstract}
The present study forms an investigation of wake breakdown in hover using the HMB3 solver. Simulations are performed for the PSP rotor blade in hover on a grid aimed at resolving the detailed flow structures in the rotor wake. An assessment of different solver settings is performed including time discretisation, spatial discretisation accuracy and turbulence modelling on the rotor wake resolution and formation of instabilities. A particular focus is put on the existence and resolution of $\mathrm{S}$-shaped structures due to the interactions of the blade tip vortices with the shear layers.
\end{abstract}

\section{Introduction}

The accuracy of hover performance predictions is of paramount importance for many vertical lift aircraft. Many low fidelity methods such as actuator disk theory or blade element momentum theory are seen to have a major drawback - a lack of modelling of the blade-vortex interaction and the effect of the rotor wake. Resolving the helical structure of the rotor wake as seen by Gray [1] is seen to be a key component of a good hover solution. The rotor wake resolution will impact the rotor downwash field, and hence the induced power prediction. Modern CFD solutions need to accurately capture the tip vortex roll up process and the wake convection for accurate performance predictions. While the far-field wake does not have a significant impact on the rotor performance, for an accurate numerical method, the physics need to be resolved correctly. For this reason, the phenomenon of wake breakdown has received particular attention in literature [2-4], to distinguish whether the wake flow structure instabilities are physical or numerical. Recent experiments confirm the presence of S-shaped structures between the individual blade tip vortices [5], however, many CFD solutions show the formation of secondary braid instabilities that overload the entire solution, which are not likely to be physical. The nature of CFD solutions is a constant challenge between numerical and physical dissipation. Many hovering solutions till date, are performed either on too coarse grids to resolve such phenomena or exhibit damping mechanisms within the computational setup (e.g grid stretching) and/or turbulence modelling approaches. The investigation of the correct approaches in the treatment of turbulent rotor wakes is one of the key aspects furthering the current state of the art of hovering rotor simulations. In the present work, we aim to investigate the modelling sensitivities associated with predictions of wake instabilities using the HMB3 solver.

\footnotetext{
*Research Assistant, CFD Laboratory, School of Engineering, Email: thomas.fitzgibbon@ glasgow.ac.uk

†Professor, MAIAA, MRAeS, CFD Laboratory, School of Engineering, Email: George.Barakos@ glasgow.ac.uk
} 


\section{HMB3 Solver}

The Helicopter Multi-Block (HMB) [6, 7] code is used as the CFD solver for the present work. It solves the Unsteady Reynolds Averaged Navier-Stokes (URANS) equations in integral form using the Arbitrary Lagrangian Eulerian (ALE) formulation for time-dependent domains, which may include moving boundaries. The Navier-Stokes equations are discretised using a cell-centred finite volume approach on a multi-block grid. The spatial discretisation of these equations leads to a set of ordinary differential equations in time,

$$
\frac{d}{d t}\left(\boldsymbol{W}_{i, j, k} V_{i, j, k}\right)=-\boldsymbol{R}_{i, j, k}(\boldsymbol{W})
$$

where $i, j, k$ represent the cell index, $\boldsymbol{W}$ and $\boldsymbol{R}$ are the vector of conservative flow variables and flux residual respectively, and $V_{i, j, k}$ is the volume of the cell $i, j, k$. To evaluate the convective fluxes, the Osher [8] approximate Riemann solver is used, while the viscous terms are discretised using a second order central differencing spatial discretisation. The Monotone Upstream-centred Schemes for Conservation Laws, which is referred to in the literature as the MUSCL approach and developed by Leer [9], is used to provide high-order accuracy in space. The HMB solver uses the alternative form of the Albada limiter [10] being activated in regions where a large gradients are encountered mainly due to shock waves, avoiding the non-physical spurious oscillations. An implicit dual-time stepping method is employed to performed the temporal integration, where the solution is marching in pseudo-time iterations to achieve fast convergence, which is solved using a first-order backward difference. The linearised system of equations is solved using the Generalised Conjugate Gradient method with a Block Incomplete Lower-Upper (BILU) factorisation as a pre-conditioner [11]. To allow an easy sharing of the calculation load for parallel job, a multi-block structured meshes are used. For the RANS/URANS calculations the $k-\omega$ SST turbulence model is used.

For the investigation of wake breakdown in hover, various features of the HMB3 solver are utilised to reduce the amount of damping in the solution. Firstly, a 4th order MUSCL scheme is used for better preservation of the blade tip vortices, implemented in the HMB3 solver by Jimenez and Barakos [12]. The scheme requires second order derivatives which are evaluated using a successive application of Green-Gauss with a minimal memory overhead of $23 \%$. Currently, the MUSCL4 scheme is only applied to the inviscid fluxes with 2nd order evaluation of the viscous fluxes and is only activated on the background grid. Secondly, the Scale Adaptive Simulation (SAS) clip is used to reduce the high levels of turbulent eddy viscosity in the rotor wake seen in many RANS hover solutions [13]. The $k-\omega$ SST SAS model, leads to an additional source term in the $\omega$ equation which is sensitive to resolved unsteady fluctuations. The source term adjusts the turbulent length scale to local flow inhomogeneities, balancing the contributions of the modelled and resolved parts of the turbulent stress tensor. The model recovers a RANS solution in stationary flows, and reduces the eddy viscosity according to the locally resolved vortex size represented by the von Karman length scale for flows with transient instabilities. 


\section{Geometry}

The four-bladed PSP rotor has an aspect ratio $(R / c)$ of 12.2 and a nominal twist of -14 degrees. The main characteristics of the rotor blades are summarised in Table 1 The blade planform has been generated using three radial stations. First, the RC(4)-12 aerofoil was used up to $65 \% R$. Then, the RC(4)-10 aerofoil from $70 \% R$ to $80 \% R$. Finally, the RC(6)-08 aerofoil was used from $85 \% \mathrm{R}$ to the tip. The aerodynamic characteristics of these aerofoils can be found in [14, 15]. The planform of the PSP model rotor has a $60 \%$ tapered and $30^{\circ}$ swept tip with the planform geometry shown in Figure 1 .

Table 1 Geometric properties of the PSP rotor blade.

\begin{tabular}{lc}
\hline \hline \multicolumn{1}{c}{ Parameter } & Value \\
\hline Number of blades $\left(N_{\mathrm{b}}\right)$ & 4 \\
Rotor radius $(R)$ & 66.50 inches \\
Rotor blade chord $(c)$ & 5.45 inches \\
Aspect ratio $(R / c)$ & 12.2 \\
Rotor solidity $(\sigma)$ & 0.1033 \\
Linear twist angle $(\Theta)$ & $-14^{\circ}$ \\
\hline \hline
\end{tabular}

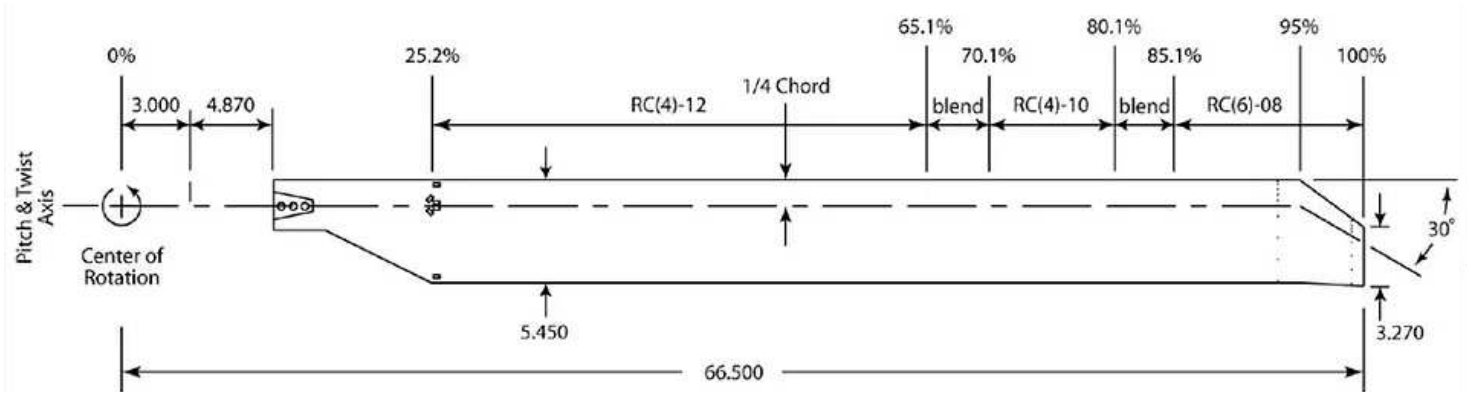

Fig. 1 Geometry of the PSP planform [16].

\section{Grids and Computational Setup}

The simulations for this study are performed on a finer grid than used for typical hover performance engineering calculations [17]. The chimera technique is used, with all four blades modelled. A single PSP blade grid is meshed using 7.2 million cells (28.8 million cells for 4 blades), with the root cutout neglected. The first background level a Cartesian grid and is set to a cell size of 5\%c (chord) leading to a grid size of 122 million cells. The background is then slowly coarsened through intermediate background grids leading to a total grid size of 158 million cells. The details of the grids are shown in Table 2 The grid setup is focused on accurately capturing the rotor blade wake up to 1.5 rotor radii, as shown in Figure 2. The simulation conditions for the wake breakdown study are shown in Table 3

The wake breakdown investigation started from a steady hover simulation with four blades and Froude boundary 
Table 2 Details of the fine PSP hover grid used for the wake breakdown study.

\begin{tabular}{ll}
\hline \hline Blade grid & \\
\hline Wall distance & $5.0 \cdot 10^{-6} c_{\text {ref }}$ \\
\hline Points along the span & 196 \\
Points around the aerofoil & 250 \\
Points normal to blade & 74 \\
Chimera extent & $0.75 \mathrm{c}$ \\
Total size (millions of cells) & 7.2 million \\
\hline Background grid & \\
Cell size of first level & $5 \% \mathrm{c}$ \\
Extent of first level in normal direction & $+1.5 \mathrm{c}$ to $-19.5 \mathrm{c}$ \\
Extent of first level in radial direction & $13.5 \mathrm{c}$ \\
Total size of first level (millions of cells) & 122 million \\
Number of intermediate background grids & 7 \\
Cell size of outer background grid & $640 \% \mathrm{c}$ \\
Extent of outer background grid & $256 \mathrm{c}$ by $256 \mathrm{c}$ by $256 \mathrm{c}$ \\
Total mesh size with blades & 158 million cells \\
\hline
\end{tabular}

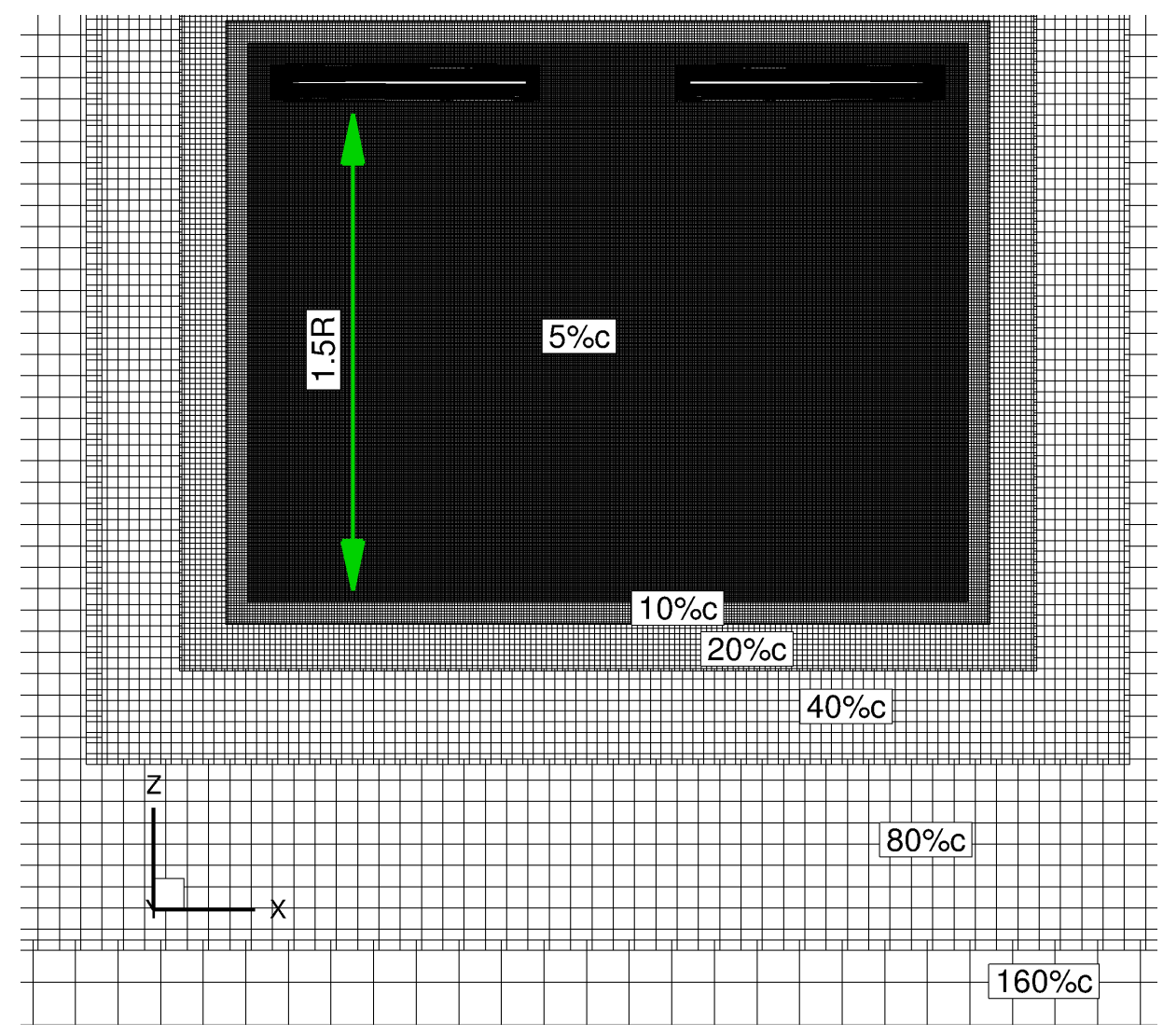

Fig. 2 Hover grid for the PSP rotor blade aimed at resolving the detailed structures in the rotor wake. 
Table 3 Simulation conditions for the wake breakdown study of the PSP rotor blade in hover.

\begin{tabular}{cc}
\hline \hline Parameter & Value \\
\hline Rotor collective, $\theta_{0}$ & $10^{\circ}$ \\
Rotor coning, $\beta_{0}$ & $0^{\circ}$ \\
Blade tip Mach number & 0.65 \\
Blade tip Reynolds number & $1.26 \times 10^{6}$ \\
\hline \hline
\end{tabular}

conditions. This was done to reduce the computational costs of unsteady hover simulations which require a number of revolutions before the rotor wake develops. Four blades were used as opposed to one as study in literature indicated that periodic boundary conditions can have a damping effect on the overall solution [2]. The steady simulation was performed for 100,000 iterations, ensuring that the rotor wake connects with the outflow Froude boundary condition. Before switching to an unsteady simulation the MUSCL 3rd order scheme was switched to 4th order and the solution was continued for an additional 80,000 iterations. An unsteady RANS simulation was then launched from the steady solution. As the steady solution was obtained in the non-inertial reference frame, no correction was required to the grid velocities in the unsteady simulation, as the background grid was rotated together with the rotor blades. The freestream boundary condition was also switched from Froude to farfield. The URANS simulation was performed for four rotor revolutions at a 1 degree time step. Finally, the time step was reduced to 0.25 degree, and the SAS-clip was switched on to reduce the turbulent eddy viscosity ratio in the rotor wake. The SAS simulation was performed for 3 rotor revolutions.

\section{Investigation of Wake Breakdown - Results}

The present section is aimed at evaluating the sensitivity of different computational setups and turbulence models on the hover solution with a focus on analysing the rotor wake. Firstly, the integrated loads are compared. Next, the wakes are compared from the RANS and SAS solutions using visualisations of Q-criterion along with a comparison of the turbulence eddy viscosity levels.

\section{A. Integrated Blade Loads}

Firstly, the integrated loads are compared in terms of the solver order (MUSCL3 and MUSCL4) and using the steady vs unsteady formulation. The results are shown in Table4

As can be seen in Table 4 the solver order or treatment of time discretisation has a minor impact on the integrated loads. The 4th order unsteady solution has a slightly higher thrust and torque compared to the steady 3rd order solution, with a similar value of Figure of Merit. All four cases predict a Figure of Merit within 1 count. 


\begin{tabular}{lccc}
\hline \hline Simulation & $C_{T} / \sigma$ & $C_{Q} / \sigma$ & FoM \\
\hline RANS, 3rd order & 0.0827 & 0.00774 & 0.698 \\
RANS, 4th order & 0.0836 & 0.00777 & 0.707 \\
URANS $\left(\Delta \psi=1^{\circ}\right)$, 4th order & 0.0844 & 0.00795 & 0.701 \\
SAS $\left(\Delta \psi=0.25^{\circ}\right), 4$ th order & 0.0838 & 0.00783 & 0.704 \\
\hline
\end{tabular}

Table 4 Integrated loads for the PSP rotor blade at $\theta_{10}=10^{\circ}$ for the RANS/URANS/SAS cases with varying solver order.

\section{B. Wake visualisation}

The wakes are visualised for each of the cases using Q-criterion, coloured by downwash velocity. Firstly, the steady and unsteady RANS cases are presented. Following this, the scale-resolving SAS cases are analysed. Slices of Q-criterion for the RANS simulations are shown in Figure 3, whereas the wake visualisation is shown in Figure 4.

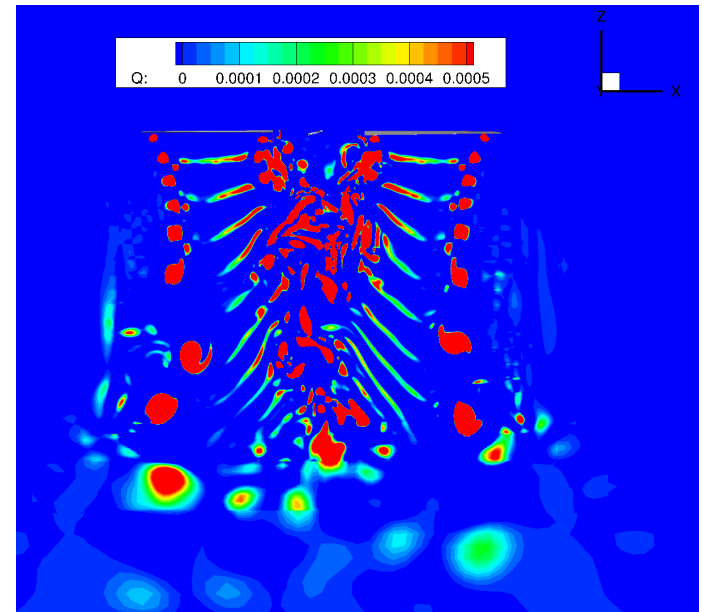

(a) Steady, 3rd order

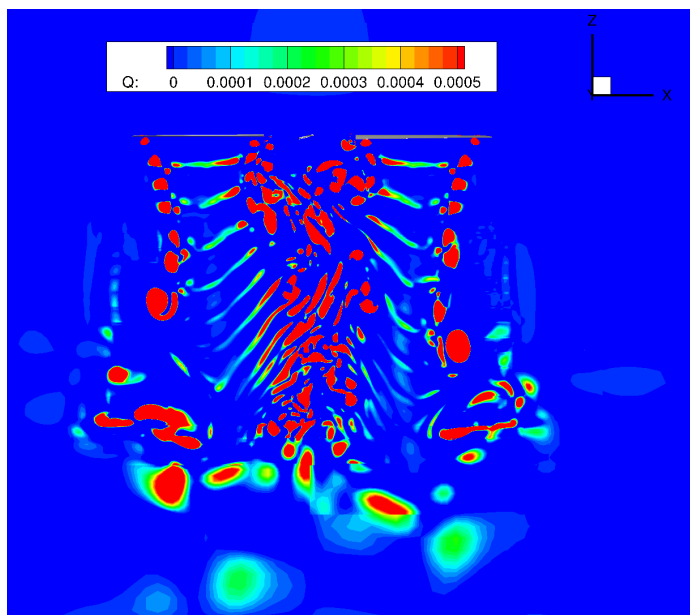

(b) Steady, 4th order

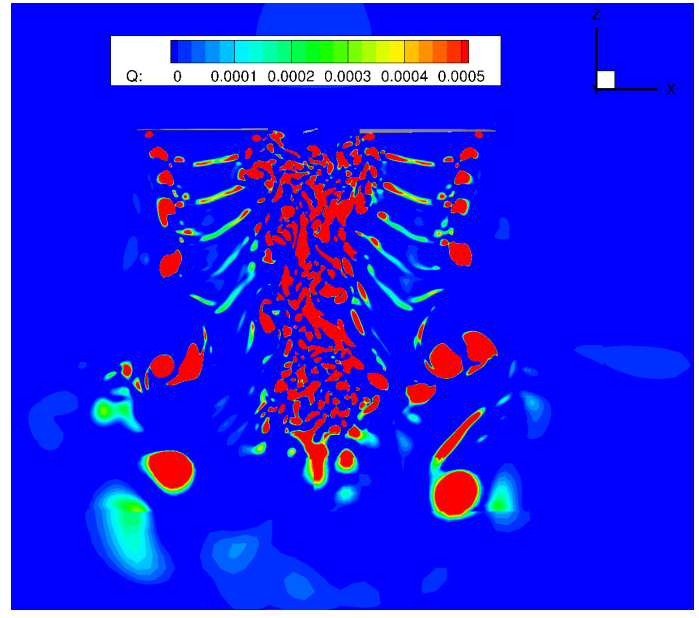

(c) Unsteady, 4th order

Fig. 3 Contours of Q-criterion at a slice at $y=0$ based on 3/4th order MUSCL extrapolation and steady/unsteady time discretisation for the PSP rotor blade in hover using a RANS-based turbulence model. 


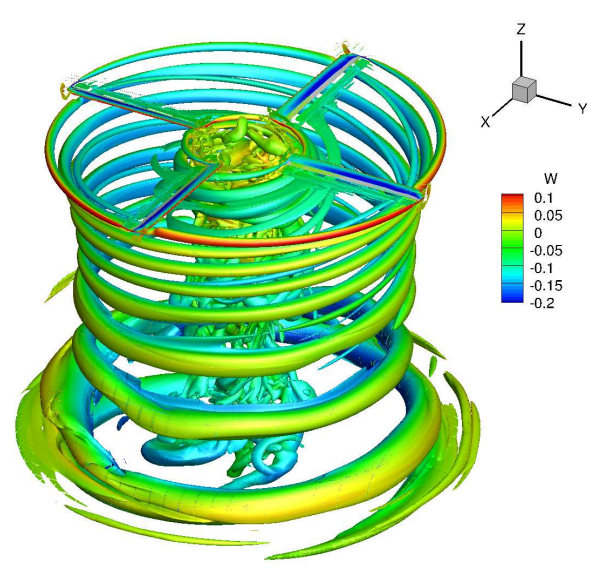

(a) Steady, 3rd order

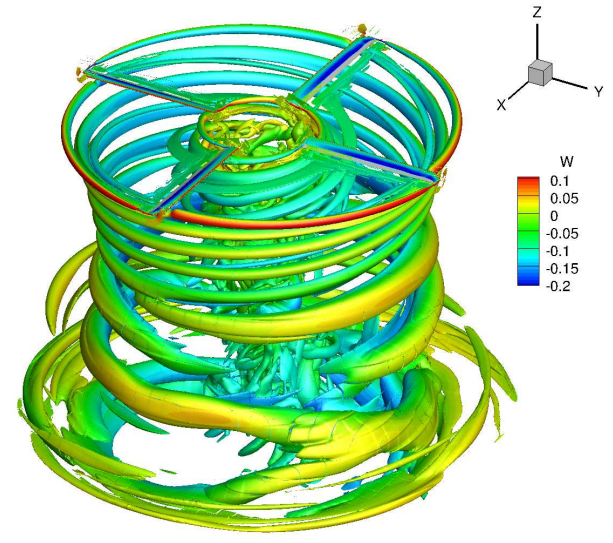

(b) Steady, 4th order

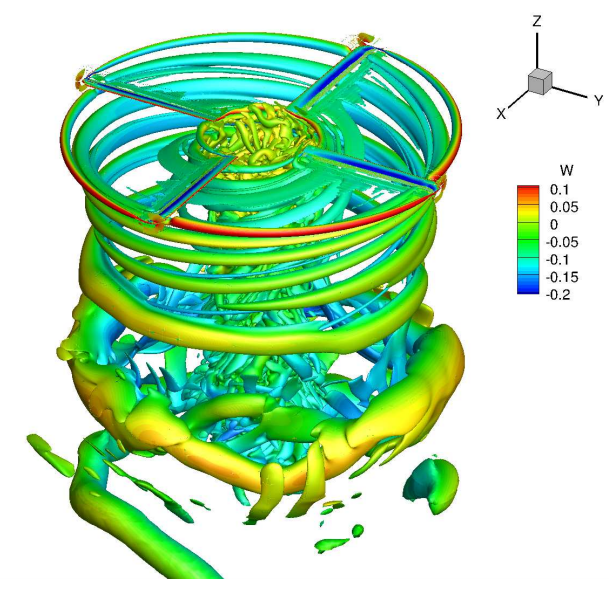

(c) Unsteady, 4th order

Fig. 4 Isosurfaces of Q-criterion (value of 0.001 ) coloured by downwash velocity based on 3/4th order MUSCL extrapolation and steady/unsteady time discretisation for the PSP rotor blade in hover using a RANS-based turbulence model.

The RANS based solutions were simulated first, due to lower computational costs and lower complexity. The slices of Q-criterion at $\mathrm{y}=0$ show highly similar behaviour, with coherent vortices in the upper wake region and greater disorder in the lower wake region, where the vortices interact with each other and the shear layers. However, no clear evidence of the presence S-shaped structures can be seen from the RANS solutions, based on the Q-criterion isosurfaces. The 4th order solution does not lead to significant differences when compared with the 3rd order solution, hence, the grid resolution is already sufficient enough to resolve the appropriate structures. The non-resolution of the S-shaped structures is hence likely to be due to the turbulence modelling approach rather than grid resolution/solver order. Moving to an unsteady solver, also does not greatly change the structure of the wake. A greater instability is seen in the lower wake, with finer detailed structures below the root of the blades, without the presence of S-shaped vortical structures or wake breakdown. The RANS based solutions lead to high turbulence eddy-viscosity levels in the rotor wake as can be seen in Figure 5 


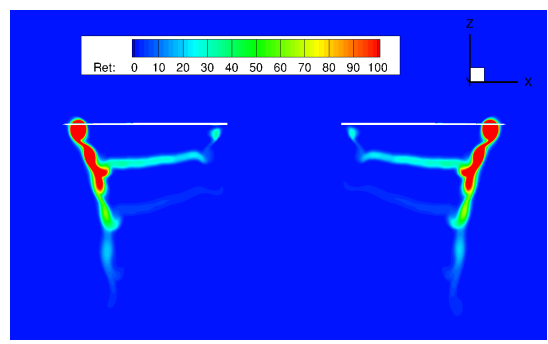

(a) Steady, 3rd order

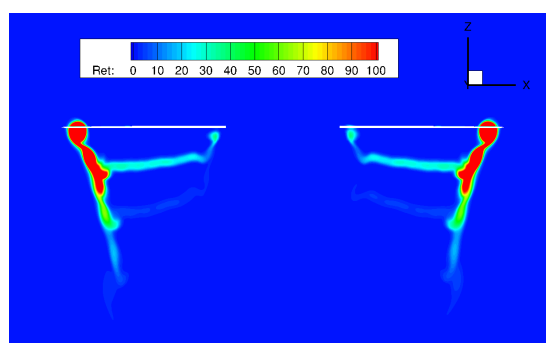

(b) Steady, 4th order

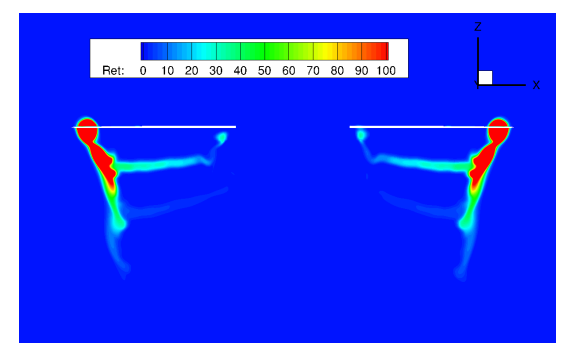

(c) Unsteady, 4th order

Fig. 5 Contours of turbulent eddy viscosity ratio at $\mathbf{y}=0$ based on RANS type simulations.

The turbulence eddy viscosity is in the order of 100's in the wake, leading to additional damping that may suppress flow instabilities or the formation more detailed wake structures. For this reason, the decision was taken to move to scale-resolving simulations, aimed to reducing the levels of turbulent eddy viscosity in the wake. The SAS simulation was restarted from the URANS case with a 4th order MUSCL scheme and 0.25 degree time step. The rotor wake is visualised over three revolutions in Figures 6.7

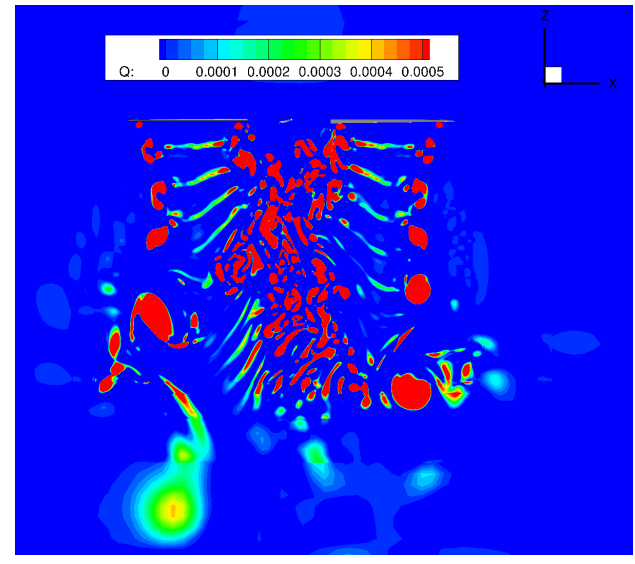

(a) 1 st revolution

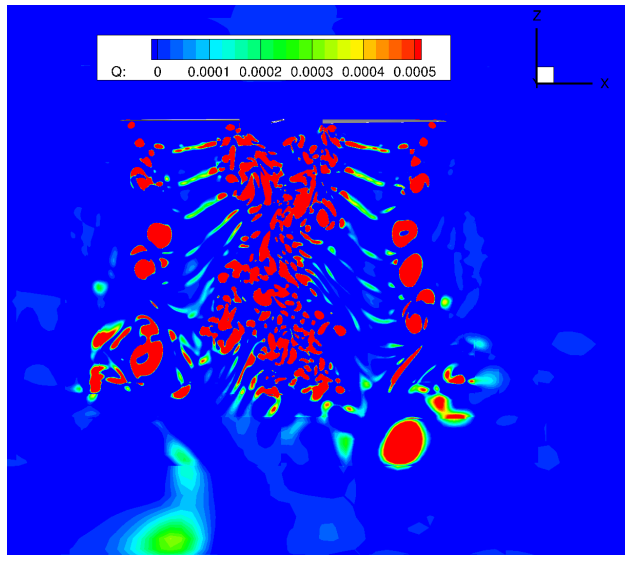

(b) 2nd revolution

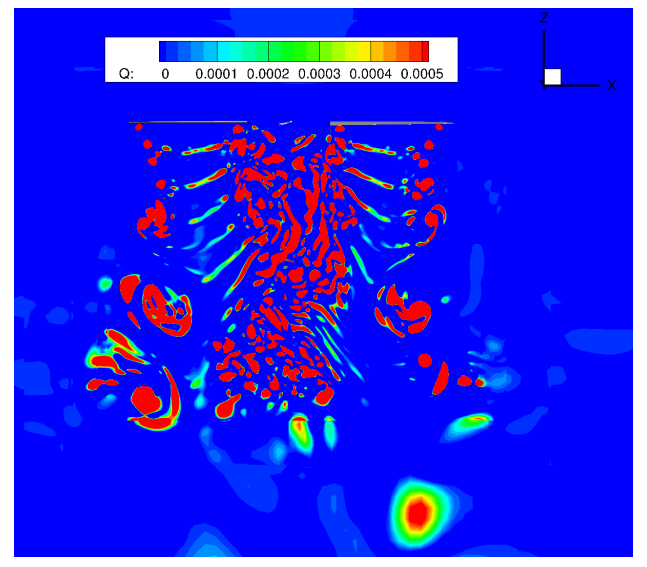

(c) 3rd revolution

Fig. 6 Contours of $\mathrm{Q}$-criterion at a slice at $\mathrm{y}=0$ with increasing revolution, based on a SAS simulation using 4th order MUSCL and 0.25 degree time-steps for the PSP rotor blade in hover. 


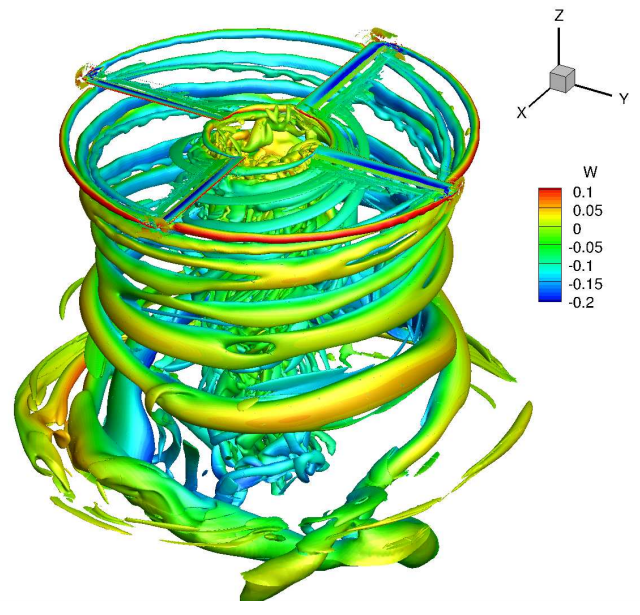

(a) 1 st revolution

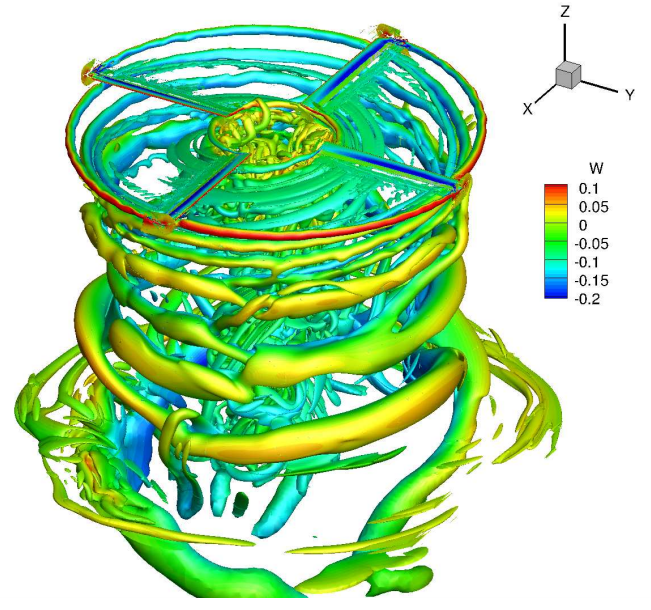

(b) 2nd revolution

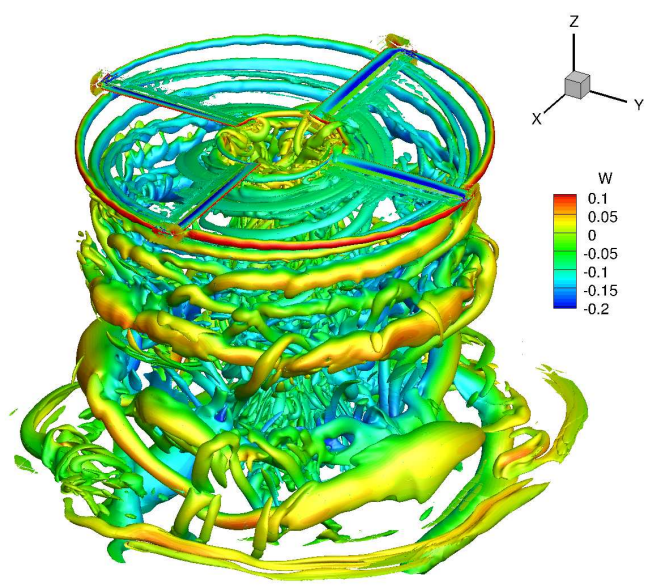

(c) 3rd revolution

Fig. 7 Isosurfaces of Q-criterion (value of 0.001 ) coloured by downwash velocity with increasing revolution, based on a SAS simulation using 4th order MUSCL and 0.25 degree time-steps for the PSP rotor blade in hover.

The high-order finer time step SAS simulations show a greater evidence of S-shaped structures and wake breakdown compared to the URANS simulations. Initially, the wake shows highly coherent blade tip vortices with little interaction of the shear layers with the rotor blade tip vortices. As the simulation progresses tip vortex pairing can be observed in the lower wake. A wide degree of finer structures is also seen further inboard in the blade root region. By the third revolution, the wake exhibits a higher degree of secondary structures with more irregularly formed blade tip vortices. Some evidence of S-shaped like wake structures can be seen in the wake visualisations. As the simulation progress, it is clear that the wake shows a less coherent structure potentially indicating the start of wake breakdown. The SAS simulation result is analysed further by looking at a contour of entropy as suggested by [18], shown in Figure 8.

The contours of entropy show a clear interaction of the shear layers with the blade tip vortices. The upper rotor wake remains fairly structured up to three vortex passages. At the fourth vortex passage, the rotor blade tip vortices start to pair with multiple interactions with the shear layers that start to curl round the main blade tip vortices. The 


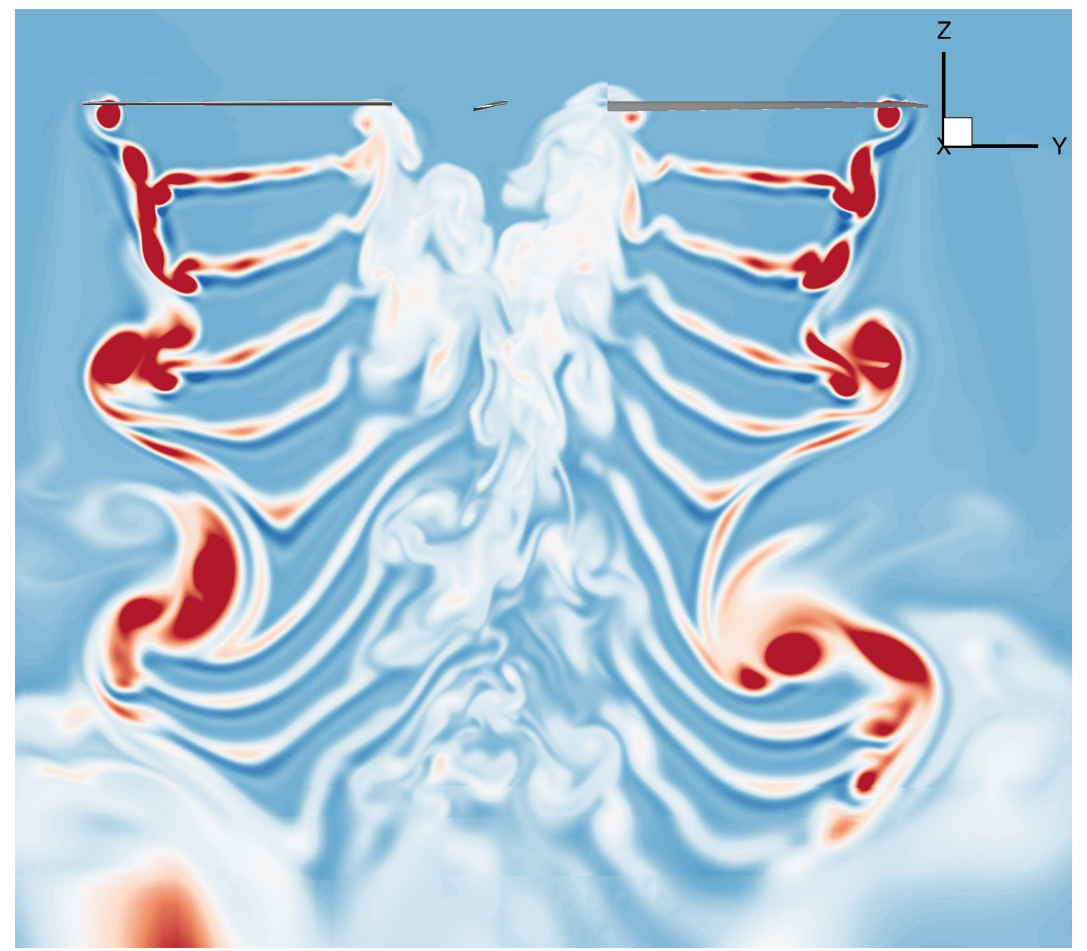

Fig. 8 Contour of entropy after 3 rotor revolutions at a slice at $y=0$, based on a SAS simulation using 4th order MUSCL and 0.25 degree time-steps for the PSP rotor blade in hover.

lower wake remains less structured although complete wake breakdown is still not present, as the blade tip vortices are still visible.

\section{Conclusions}

The wake breakdown study showed that scale-resolving simulations are required to examine the flow physics of rotor wakes. RANS based solutions lead to too high turbulent length scales in the rotor wake and hence dampen out any secondary flow structures, leading to a highly coherent wake with well formed blade tip vortices. The addition of the SAS clip shows clear evidence of secondary wake structures and a potential wake instability. As the simulation progresses the wake structure becomes less coherent, although further revolutions are required to obtain full wake breakdown which is seen as part of future work. Additionally, different turbulence modelling approaches will also be examined in future studies.

\section{Acknowledgements}

This work is funded by DSTL (Defence Science and Technology Laboratory), Project No. 74260. This work used the Cirrus UK National Tier-2 HPC Service at EPCC (http://www.cirrus.ac.uk). This work used the ARCHER UK National Supercomputing Service (http://www.archer.ac.uk). 


\section{References}

[1] Gray, R., "An Aerodynamic Analysis of a Single-Bladed Rotor in Hovering and Low-Speed Forward Flight as Determined from Smoke Studies of the Vorticity Distribution in the Wake,” Tech. rep., Princeton Univ. Aeronautical Eng. Dept., 1956. Report No. 356.

[2] Hariharan, N., Abras, J., and Narducci, R., "Wake Breakdown of High-fidelity Simulations of a Rotor in Hover," AIAA SciTech Forum, 57th Aerospace Sciences Meeting, San Diego, CA, USA, 2019.

[3] Hariharan, N., "An Overview of Wake-Breakdown in High-Fidelity Simulations of Rotor in Hover," AIAA SciTech Forum, 58th Aerospace Sciences Meeting, Orlando, FL, USA, 2020.

[4] Yoon, S., nad TH. Puliam, N. C., and Holst, T., "Effect of Turbulence Modeling on Hovering Rotor Flows," AIAA Aviation, 45th AIAA Fluid Dynamics Conference, Dallas, TX, USA, 2015.

[5] Wolf, C., Schwarz, C., Kaufmann, K., Gardner, A., Michaelis, D., Bosbach, J., Schanz, D., and Schröder, A., "Experimental study of secondary vortex structures in a rotor wake," Experiments in Fluids, Vol. 60, No. 175, 2019. DOI: 10.1007/s00348019-2807-1.

[6] Steijl, R., Barakos, G. N., and Badcock, K., "A framework for CFD analysis of helicopter rotors in hover and forward flight," International Journal for Numerical Methods in Fluids, Vol. 51, No. 8, 2006, pp. 819-847. DOI: 10.1002/d.1086.

[7] Steijl, R., and Barakos, G. N., "Sliding mesh algorithm for CFD analysis of helicopter rotor-fuselage aerodynamics," International Journal for Numerical Methods in Fluids, Vol. 58, No. 5, 2008, pp. 527-549. DOI: 10.1002/d.1757.

[8] Osher, S., and Chakravarthy, S., "Upwind schemes and boundary conditions with applications to Euler equations in general geometries," Journal of Computational Physics, Vol. 50, No. 3, 1983, pp. 447-481. DOI: 10.1016/0021-9991(83)90106-7.

[9] van Leer, B., “Towards the ultimate conservative difference scheme. V.A second-order sequel to Godunov's Method,” Journal of Computational Physics, Vol. 32, No. 1, 1979, pp. 101-136. DOI: 10.1016/0021-9991(79)90145-1.

[10] van Albada, G. D., van Leer, B., and Roberts, W. W., "A Comparative Study of Computational Methods in Cosmic Gas Dynamics," Astronomy and Astrophysics, Vol. 108, No. 1, 1982, pp. 76-84.

[11] Axelsson, O., Iterative Solution Methods, Cambridge University Press: Cambridge, MA, 1994.

[12] Jimenez-Garcia, A., and Barakos, G., "Assessment of a high-order MUSCL method for rotor flows," International Journal for Numerical Methods in Fluids, Vol. 87, No. 6, 2018, pp. 292-327. DOI: 10.1002/fld.4492.

[13] Postdam, M., and Puliam, T., “Turbulence Modeling Treatment for Rotorcraft Wakes," AHS Specialist's Conference on Aeromechanics, San Francisco, CA, USA, 2008.

[14] Noonan, K. W., “Aerodynamic Characteristics of Two Rotorcraft Airfoils Designed for Application to the Inboard Region of a Main Rotor Blade,” NASA TP-3009, U.S. Army Aviation Systems Command, TR-90-B-005, Jul. 1990. 
[15] Noonan, K. W., "Aerodynamic Characteristics of Two Rotorcraft Airfoils Designed for Application to the Inboard Region of a Main Rotor Blade,” NASA TM-4264, U.S. Army Aviation Systems Command, TR-91-B-003, May 1991.

[16] Committee, A. A. A. T., "PSP Rotor Description," https://www.aiaa-hpw.org/psp-rotor, 2021. [Online; accessed 30-November-2021].

[17] T. Fitzgibbon, A. J., MA. Woodgate, and Barakos, G., "Validation of the Steady-State Hover Formulation for AccuratePerformance Predictions," AIAA Journal, Vol. 57, No. 12, 2019, pp. 5293-5308. DOI: 10.2514/1.J058408.

[18] Hariharan, N., Abras, J., and Narducci, R., "Impact of High-fidelity Simulation Variations on Wake Breakdown of a Rotor in Hover," AIAA SciTech Forum, 58th Aerospace Sciences Meeting, Orlando, FL, USA, 2020. 